\title{
Endometrial Carcinoma
}

National Cancer Institute

\section{Source}

National Cancer Institute. Endometrial Carcinoma. NCI Thesaurus. Code C7558.

A malignant tumor arising from the epithelium that lines the cavity of the uterine body.

The vast majority of endometrial carcinomas are adenocarcinomas; squamous cell and adenosquamous carcinomas represent a minority of the cases. Endometrioid adenocarcinoma is the most frequently seen variant of endometrial adenocarcinoma. Uterine bleeding is an initial clinical sign. The prognosis depends on the stage of the tumor, the depth of myometrial wall invasion, and the degree of differentiation. 